1 The Consequences of Egg Adaptation in the H3N2 Component to the Immunogenicity of

\title{
2 Live Attenuated Influenza Vaccine
}

3

4 Daniel H. Goldhill ${ }^{1}$, Benjamin Lindsey ${ }^{1,2}$, Ruthiran Kugathasan ${ }^{1}$, Zandra Felix Garza ${ }^{3}$, Ya

5 Jankey Jagne ${ }^{2}$, Hadijatou Jane Sallah² ${ }^{2}$, Gabriel Goderski ${ }^{4}$, Sophie van Tol ${ }^{4}$, Katja Höschler ${ }^{5}$,

6 Adam Meijer ${ }^{4}$, Wendy S. Barclay ${ }^{1}$ and Thushan I. de Silva ${ }^{1,2,6}$.

7

1. Department of Infectious Disease, Imperial College London, St Mary's Campus, London, W2 1PG, UK.

2. Vaccines and Immunity Theme, Medical Research Council Unit The Gambia at the London School of Hygiene and Tropical Medicine, Atlantic Road, PO Box 273, Banjul, The Gambia

3. Department of Medical Microbiology, Academic Medical Center, University of Amsterdam,

4. Centre for Infectious Disease Research, Diagnostics and Laboratory Surveillance, National Institute for Public Health and the Environment, Antonie van Leeuwenhoeklaan 9, $3721 \mathrm{MA}$ Bilthoven, The Netherlands.

5. Virus Reference Department, Reference Microbiology Services, Public Health England, Colindale Avenue, London NW9 5HT, UK.

6. The Florey Institute for Host-Pathogen Interactions and Department of Infection, Immunity and Cardiovascular Disease, University of Sheffield, Medical School, Sheffield S10 2RX, UK.

22 Corresponding author: Thushan de Silva: tdesilva@mrc.gm, +44 7976605320

Keywords: Live Attenuated Influenza Vaccine, H3N2, Egg-adaptation, Primer ID

Word count: 1999/2000. Brief Report. 


\section{Footnote Page}

\section{Conflicts of Interest}

28 The authors have no conflicts of interest.

\section{$29 \quad$ Funding}

30 This work was supported by a Wellcome Trust Intermediate Clinical Fellowship award to TdS

$31(110058 / \mathrm{Z} / 15 / \mathrm{Z})$ and by a Biotechnology and Biological Sciences Research Council grant

$32(\mathrm{BB} / \mathrm{K} 002465 / 1)$ and a Wellcome grant $(205100 / \mathrm{Z} / 16 / \mathrm{Z})$ to WSB. RK was supported by

33 Wellcome fellowship (216353/Z/19/Z).

34

35 Portions of this work were previously presented as a poster at Options X in Singapore in 2019.

36 Abstract Number: 11111.

37

38 Corresponding author: Thushan de Silva: tdesilva@mrc.gm, +44 7976605320. Department 


\section{Abstract}

45 Adaptation in egg-passaged vaccine strains may cause reduced vaccine effectiveness due to

46 altered antigenicity of the influenza haemagglutinin. We tested whether egg adaptation

47 modified serum and mucosal antibody responses to the $A(H 3 N 2)$ component in the Live

48 Attenuated Influenza Vaccine (LAIV). Twice as many children seroconverted to an egg-

49 adapted H3N2 than the equivalent wildtype strain. Seroconversion to the wildtype strain

50 was greater in children seronegative pre-LAIV, whereas higher mucosal IgA responses to

51 wildtype antigen were observed if seropositive prior to vaccination. Sequencing of virus

52 from nasopharyngeal swabs from 7 days post-LAIV showed low sequence diversity and no

53 reversion of egg-adaptive mutations.

54 Word count: $100 / 100$

55 
58 Vaccines offer the best protection against morbidity and mortality caused by influenza. Low vaccine effectiveness (VE) is often attributed to an antigenic mismatch between the vaccine and circulating strains caused by evolution of the influenza haemagglutinin (HA) [1]. component was reported, potentially due to adaptive mutations caused by the production of the influenza vaccine in eggs [1]. These egg-adaptive mutations can alter vaccine antigenicity and lead to immune responses mismatched to circulating strains.

65

Of the four components of the current quadrivalent seasonal influenza vaccine, the issue of egg adaptation resulting in antigenic mismatch is most problematic for H3N2 subtype influenza A viruses. In 2014, the H3N2 3C.2a clade arose with a K160T mutation, giving an additional putative glycosylation site in immunodominant antigenic site B on the HA head [2]. In order to grow efficiently, 3C.2a strains revert from T160 to K160 when cultured in eggs and this adaptive mutation has been suggested as a key reason for the recent loss of H3N2 VE [1]. The reduction in immunogenicity against circulating strains caused by this reversion has so far been described in the context of serum antibody responses to inactivated influenza vaccine (IIV) [1]. Here, we examine the consequence of eggadaptations in the Live Attenuated Influenza Vaccine (LAIV) on serum and mucosal antibody responses in young children to circulating T160-containing H3N2. We also sequence virus to a human-adaptive form. 
Methods

81

\section{Study design and sample collection}

The samples used in this study were generated during a larger randomised controlled trial

(ClinicalTrials.gov NCT02972957) comparing the impact of LAIV on the nasopharyngeal and 2018. A detailed description of the cohort and sampling is described elsewhere [3]. Influenza vaccine-naïve children aged 24-59 months received a single intranasal dose of the trivalent Russian-backbone LAIV (Nasovac-S, Serum Institute of India Pvt Ltd) containing the World Health Organisation recommended viruses for the Northern Hemisphere for either 2016-2017 or 2017-2018, dependent on the year of enrolment. For both vaccines, the H3N2 component was an A/Hong Kong/4801/2014 (H3N2)-like virus. H3N2 vaccine titres per dose (50\% Egg Infectious Doses (EID50)/ml) were $1 \times 10^{7.5}$ in 2017 and $1 \times 10^{7.6}$ in 2018. RNAprotect cell reagent (Qiagen) on day 2 (D2) and 7 (D7) post-vaccination. Serum and oral fluid swab (Oracol Plus; Malvern Medical Development, Worcester, UK) samples were taken on D0 and day 21 (D21). All samples were stored at $-70^{\circ} \mathrm{C}$ before further processing. The study was approved by The Gambia Government/MRC Joint Ethics Committee and the informed consent for their children to participate.

HAI and IgA assays red blood cells (0.5\% in PBS) in the absence of oseltamivir and with standard methods [4]. 
HAI titres pre- and D21 post-LAIV were determined to cell-cultured (in MDCK-SIAT cells) and egg-cultured A/Hong Kong/4801/2014 (HK14) strains. Sequencing of viral stocks confirmed the presence of T160 in cell-cultured and K160 in egg-cultured HK14. Egg-cultured virus also contained further egg-adaptive mutations L194P, T203I and I260L. Seroconversion was defined as a 4-fold rise in HAI titre to $\geq 1: 40$, irrespective of baseline HAI titre, using a 2-fold dilution series of serum starting from 1:10 dilution. Mucosal influenza-specific IgA was measured in oral fluid samples at baseline and D21 post-LAIV using a protein microarray as previously described $[5,6]$ with the percentage Surfact-Amps-20 in the blocking, washing and incubation buffer increased from $0.05 \%$ to $5 \%$ to prevent background staining with oral specimens. Microarrays were coated with recombinant HA1 protein expressed in human cells (Sino Biological, Beijing, China) reflecting sequences of egg-adapted and cell-cultured HK14. Total IgA was quantified using an ELISA and influenza-specific IgA expressed as a ratio of influenza HA1-specific IgA/total IgA as previously described [6].

\section{RNA Extraction, Primer ID and Sequencing}

119 RNA was extracted from D2 and D7 nasopharyngeal samples previously identified as positive for H3N2 shedding by RT-PCR [3], using QIAamp Viral RNA Mini Kit (Qiagen) with carrier RNA. RNA was also extracted from vaccine aliquots diluted 1000-fold. RNA was reverse transcribed using Superscript III (Invitrogen) and a barcoded primer specific to each 500bp sub-amplicon in the HA (Primer ID). Primer ID attaches a unique barcode to each CDNA molecule during reverse transcription and allows for PCR and sequencing error correction $[7,8]$. PCR was performed using KOD polymerase (Merck). Samples were pooled across sub-amplicons and prepared for sequencing using NebNext Ultra II (NEB), then 
Geneious (v11) and a pipeline in R. Forward and reverse reads were paired using FLASh

(https://ccb.jhu.edu/software/FLASH) before being mapped to a reference sequence and consensus sequences made for each barcode. Degenerate barcodes were removed (see chosen. Raw sequences were deposited at https://www.ebi.ac.uk/ena (project number PRJEB34129.) The analysis pipeline can be found at https://www.github.com/Flu1/GambiaLAIV.

\section{Statistical analysis}

137 Paired and unpaired proportions were compared using McNemar's and $\mathrm{Chi}^{2}$ tests

138 respectively. HAI geometric mean fold rise (GMFR) within and between individuals was compared using the Wilcoxon signed-rank and Mann-Whitney tests. Log10-transformed IgA fluorescence ratio fold-change was compared using paired and unpaired t-tests. Pairwise correlations were assessed using Spearman's rank-order (GMFR) and Pearson's (log10mutations within each sequenced sample (Supplementary material). Genetic distance

144 between samples was calculated as described in Supplementary material. Statistical analyses were performed using R version 3.5.0 and GraphPad Prism 8.0.2.

\section{Results}

148 Samples from 244 children were included in the HAI analysis ( $n=118$ from 2017 and $n=126$

149 from 2018 [3]). Influenza-specific oral fluid IgA data were available from 214 children ( $n=100$

150 from 2017 and $n=114$ from 2018). D7 nasopharyngeal swab samples from 30 children with 
153 No significant differences were seen between pre-LAIV HAI titres to egg-cultured and cellcultured HK14 ( $p=0.84$, Figure S2). The proportion of children who seroconverted to eggcultured HK14 virus was 25.0\% (61/244, 95\% confidence interval (CI) 19.6-30.4) compared to $12.3 \%(30 / 244,95 \% \mathrm{Cl} 8.2-16.4)$ to cell-cultured HK14 $(p<0.0001)$. D0 to D21 GMFR to significant correlation was present between GMFR to egg-cultured and cell-cultured HK14 $\left(r_{s}=0.58, p<0.0001\right.$, Figure S3), although discrepant samples were observed with seroconversion to only one virus (Figure 1B). In contrast, the increase in mucosal influenza HA-specific IgA from D0 to D21 post-LAIV was greater to cell-culture matched HK14 HA compared to egg-culture matched HK14 HA ( $p=0.0009$, Figure 1C). A significant correlation was observed between IgA fold-change to egg- and cell-cultured HK14 HA ( $r=0.69$, $\mathrm{p}<0.0001$, Figure S4).

To explore the impact of prior H3N2 infection on serum and mucosal antibody responses to HK14 in LAIV, children were stratified based on seropositivity to cell-cultured HK14 (preLAIV HAI titre $\geq 1: 10)$. In seronegative children, seroconversion to egg-cultured HK14 (50.7\%, $37 / 73,95 \% \mathrm{Cl} 39.2-62.2 \%)$ and cell-cultured HK14 (27.4\%, 20/73, 95\% Cl 17.2-37.6\%) was greater than in seropositive children (14.0\% seroconversion to egg-cultured, $24 / 171,95 \% \mathrm{Cl}$ 8.8-19.4\%, $\mathrm{p}=0.00048$ and $5.8 \%$ seroconversion to cell-cultured, $10 / 171,95 \% \mathrm{Cl} 2.3-9.4 \%$, $\mathrm{p}<0.0001$ ). This pattern was reflected in GMFR values (Figure $2 \mathrm{~A}$ ), but not in IgA responses.

173 D0 to D21 post-LAIV IgA fold change, to HK14 HA proteins representative of both eggcultured and cell-cultured HK14, was modestly higher in seropositive children compared to seronegative children (Figure 2A). 
177 To explore whether reversion of egg-adapted mutations in LAIV HK14 during

178 nasopharyngeal replication could drive responses to cell-cultured HK14, we sequenced two

179 sub-amplicons containing HA amino-acids 1-276. 20 samples with low H3N2 cycle threshold

180 (Ct) values (five D2 and fifteen D7 from sixteen individuals) were successfully amplified

181 (Supplementary material, Figure S5). No significant reversion of egg-adaptive mutations was

182 seen in any samples (Figure 2C). This included D7 samples from seven seroconverters to cell-

183 cultured HK14. Two samples showed a single sequence with P194L (<0.2\% frequency) and

184 three samples showed one or two sequences with I203T ( $<0.2 \%$ frequency). Along with position 160 , these three sites were $>99.9 \%$ identical to the vaccine across all samples. Few mutations rose to high frequency with only five mutations occurring above $5 \%$. Of these, $123 \mathrm{~L}$ was a pre-existing polymorphism present at $1 \%$ in the vaccine strain and the other four mutations were synonymous. There was no significant difference between Shannon entropy for the samples and vaccine strains (Z-test, $p=0.41$ ). In individuals with matched samples, mutations present at higher frequencies on D2 had been lost by D7 (Figure 2C). Using the relative L1-norm as a measure of genetic similarity, there was no significant difference between samples taken from the same individual and other samples ( $Z$-test, $p=0.54$, Figure S6).

Discussion

We describe, to our knowledge for the first time, the impact of egg-adaptations in a recent H3N2 3C.2a strain vaccine antigen on serum and mucosal antibody responses induced by LAIV to the equivalent human-adapted strain reflective of circulating viruses. In keeping with observations with IIV [2], serum antibody responses to cell-cultured HK14 were 
significantly lower than to the vaccine-matched egg-cultured HK14. However, a proportion

201 of children did seroconvert to cell-cultured HK14, which was most evident in children seronegative to cell-cultured HK14 prior to receiving LAIV. In the absence of prior HK14 exposure, the serum antibody response in these children may be broader and directed to antigens outside antigenic site B [2].

In contrast to serum HAl induction, IgA responses to proteins representing cell-cultured responses were also modestly higher in children who were seropositive to HK14 prior to LAIV compared to seronegative children. Therefore, in our cohort, unlike serum antibodies induced by LAIV, mucosal IgA responses may largely reflect boosting of prior responses acquired through natural infection. However, the lack of a significant IgA correlate of protection following LAIV vaccination means that the clinical relevance of this finding is uncertain. Compared to the serum HAI response, little is known about the antigenspecificity of LAIV-induced mucosal IgA responses, although some studies have suggested

215 influenza-specific IgA responses are more cross-reactive that IgG responses $[9,10]$. It is important to note that the IgA responses measured constitute binding antibody, rather than

217 functional responses, which are challenging to measure in mucosal samples. Future work could explore functional mucosal IgA responses, as well as anti-HA stalk responses which we did not assess and may provide cross-reactive responses.

221 A previous influenza human challenge study in adults has demonstrated the reversion of an egg-adapted mutation during replication in the upper respiratory tract [11]. We 
providing a potential explanation for cell-cultured HK14-specific antibody responses after

225 vaccination with an egg-adapted antigen. Sequencing of the shed virus, however, revealed

226 no changes at sites of egg adaptation and very few significant changes in the HA. The lack of Figure1

227 a K160 fitness cost in humans is perhaps unsurprising given the majority of H3N2 isolates

228 prior to 2014 contained K160. Recent studies have found low within-host diversity of virus

229 in natural influenza infections in vaccinated and unvaccinated individuals, suggesting that

230 the immune system does not put significant pressure on the influenza virus to evolve over

231 the course of an individual infection $[12,13]$. Our results agree with this and imply there is

232 little positive selection on the LAIV H3N2 HA in the nasopharynx within the first week and that reversion of egg-adaptation mutations such as K160 is unlikely.

Although egg adaptation is likely to be an important factor, increasing data suggest several factors contribute to the low VE to H3N2 observed in some years [14]. Developing alternatives to egg-based methods of vaccine production is clearly important as current vaccines may result in protective H3N2 responses only in sub-populations of individuals.

\section{Funding}

241 This work was supported by a Wellcome Trust Intermediate Clinical Fellowship award to TdS

$242(110058 / \mathrm{Z} / 15 / \mathrm{Z})$ and by a Biotechnology and Biological Sciences Research Council grant $243(\mathrm{BB} / \mathrm{K} 002465 / 1)$ and a Wellcome grant $(205100 / \mathrm{Z} / 16 / \mathrm{Z})$ to WSB. RK was supported by 244 Wellcome fellowship (216353/Z/19/Z). 
We gratefully acknowledge the study participants and parents who took part in the study. We also acknowledge the dedicated field team involved in carrying out the clinical trial. We thank Christine Carr, Monali Patel and Hirushi Rajapakse for their technical support at the Respiratory Virus Unit (PHE NIS, Colindale).

Figure 1. a) Geometric Mean Fold Rise (GMFR) to egg-cultured and cell-cultured HK14 b) which defines seroconversion.

Figure 2. a) GMFR to egg and cell-cultured HK14 comparing children seropositive and seronegative to cell-cultured (i.e. wild-type) HK14 prior to receiving LAIV. Dotted line represents 4-fold increase in HAl titre between day 0 and day 21 which defines seroconversion. b) Fold-change from day 0 to day 21 post-LAIV in HA1-specific IgA to proteins representing egg-cultured and cell-cultured HK14, comparing seropositive and seronegative children. c) Shed virus from 20 samples from either day 2 or day 7 and the vaccine from 2017 and 2018 were sequenced using Primer ID. The percentage of mutations are shown at each sequenced nucleotide position in the HA where 1 refers to the first base of the signal peptide. The sample ID and day of sample collection are shown.

\section{References}


271 glycosylation site that alters binding of antibodies elicited by egg-adapted vaccine strains.

272 Proceedings of the National Academy of Sciences 2017; 114:12578-83.

2. Chambers BS, Parkhouse K, Ross TM, Alby K, Hensley SE. Identification of hemagglutinin

274 residues responsible for H3N2 antigenic drift during the 2014-2015 influenza season. Cell reports $2015 ; 12: 1-6$.

3. Lindsey BB, Jagne YJ, Armitage EP, et al. Effect of a Russian-backbone live-attenuated

277 influenza vaccine with an updated pandemic H1N1 strain on shedding and immunogenicity

278 among children in The Gambia: an open-label, observational, phase 4 study. Lancet Respiratory Medicine 2019; 7:665-76.

4. Ellis J, Zambon M. Molecular analysis of an outbreak of influenza in the United Kingdom. European journal of epidemiology 1997; 13:369-72.

5. Koopmans M, De Bruin E, Godeke G-J, et al. Profiling of humoral immune responses to influenza viruses by using protein microarray. Clinical Microbiology and Infection 2012; 18:797-807.

6. de Silva TI, Gould V, Mohammed NI, et al. Comparison of mucosal lining fluid sampling methods and influenza-specific IgA detection assays for use in human studies of influenza

287 immunity. Journal of immunological methods 2017; 449:1-6.

7. Jabara $C B$, Jones $C D$, Roach J, Anderson JA, Swanstrom R. Accurate sampling and deep sequencing of the HIV-1 protease gene using a Primer ID. Proc Natl Acad Sci U S A 2011; 108:20166-71. virus using next-generation sequencing. Journal of virology 2019; 93:e01217-18. 
294 influenza virus induces heterosubtypic neutralising mucosal IgA antibodies in humans.

295 Vaccine 2014; 32:1897-900.

296

10. Muramatsu M, Yoshida R, Yokoyama A, et al. Comparison of antiviral activity between

$297 \operatorname{IgA}$ and IgG specific to influenza virus hemagglutinin: increased potential of IgA for heterosubtypic immunity. PLoS One 2014; 9:e85582. human challenge study reveals a selective bottleneck and only limited intrahost genetic

301 diversification. Journal of virology 2016; 90:11247-58. diversity of H3N2 influenza viruses. PLoS pathogens 2017; 13:e1006194. processes constrain the within and between host evolution of influenza virus. Elife 2018; 7:e35962. adaptation, may explain the low H3N2 influenza vaccine effectiveness in 2012-2013. Clinical Infectious Diseases 2018; 67:327-33. 


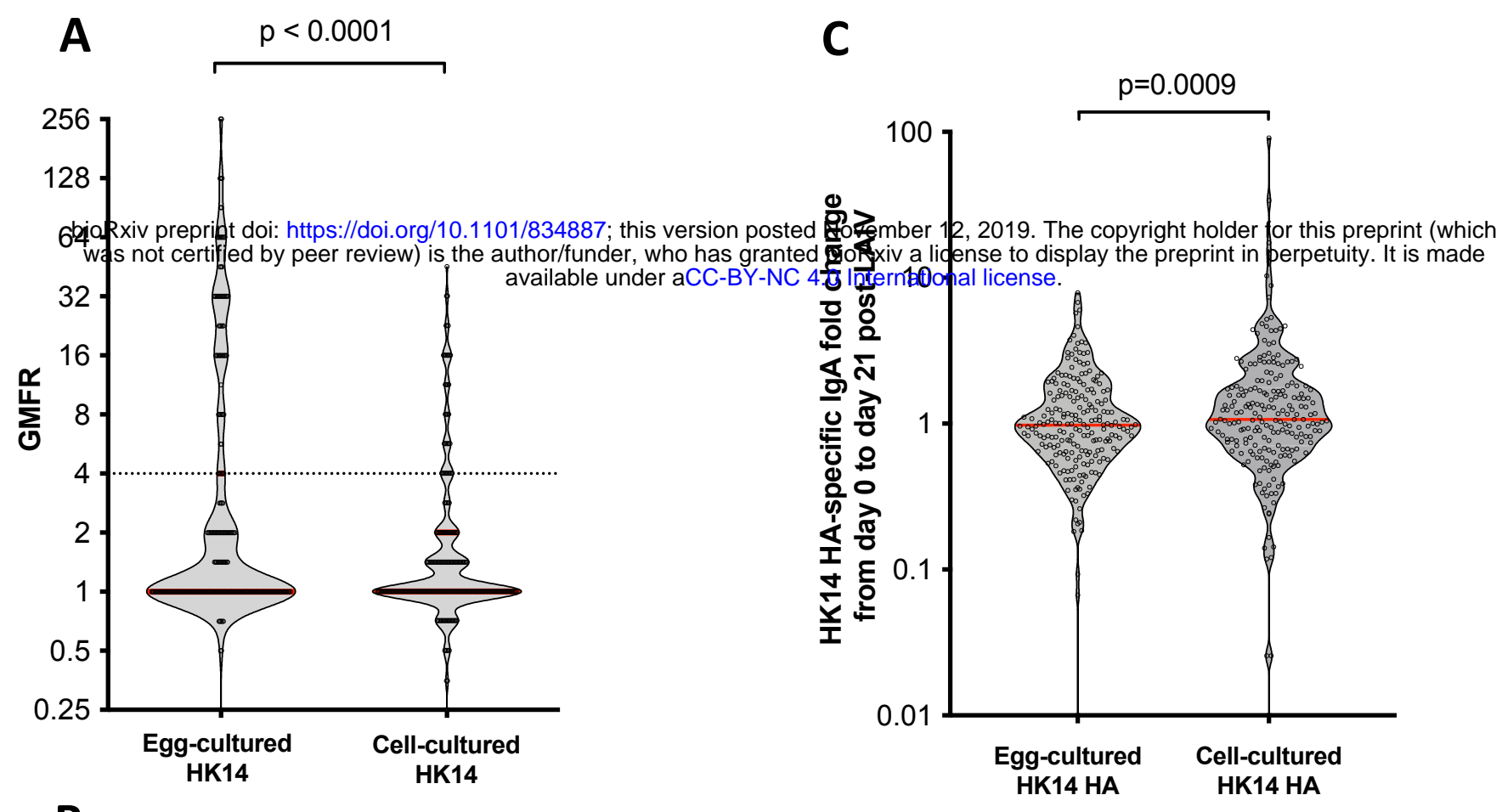

B

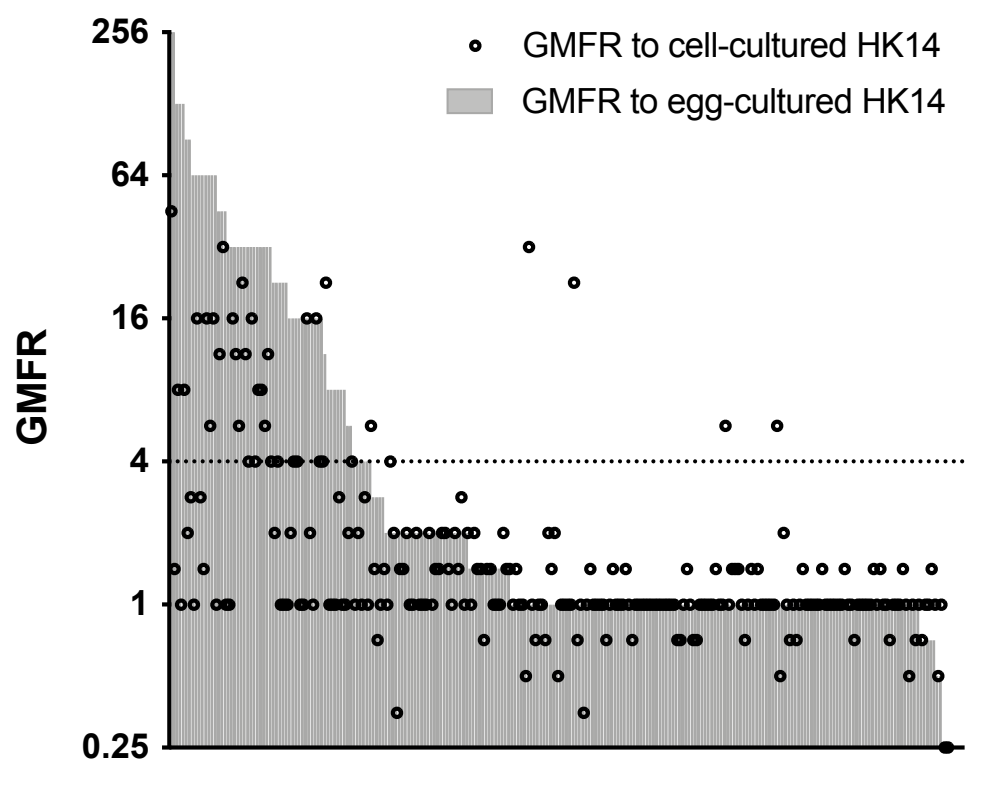


A

$p<0.0001$

$p=0.0005$

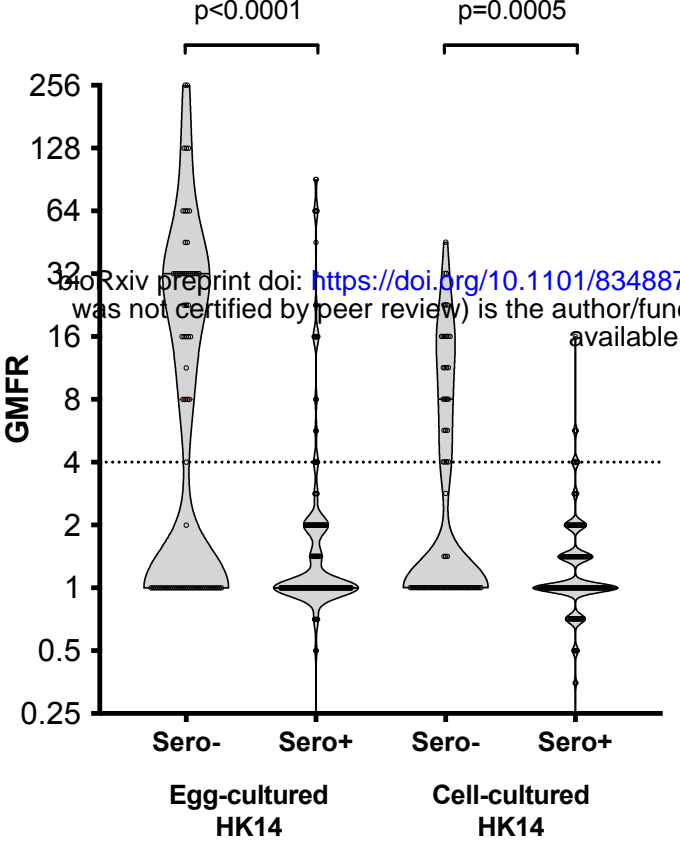

C
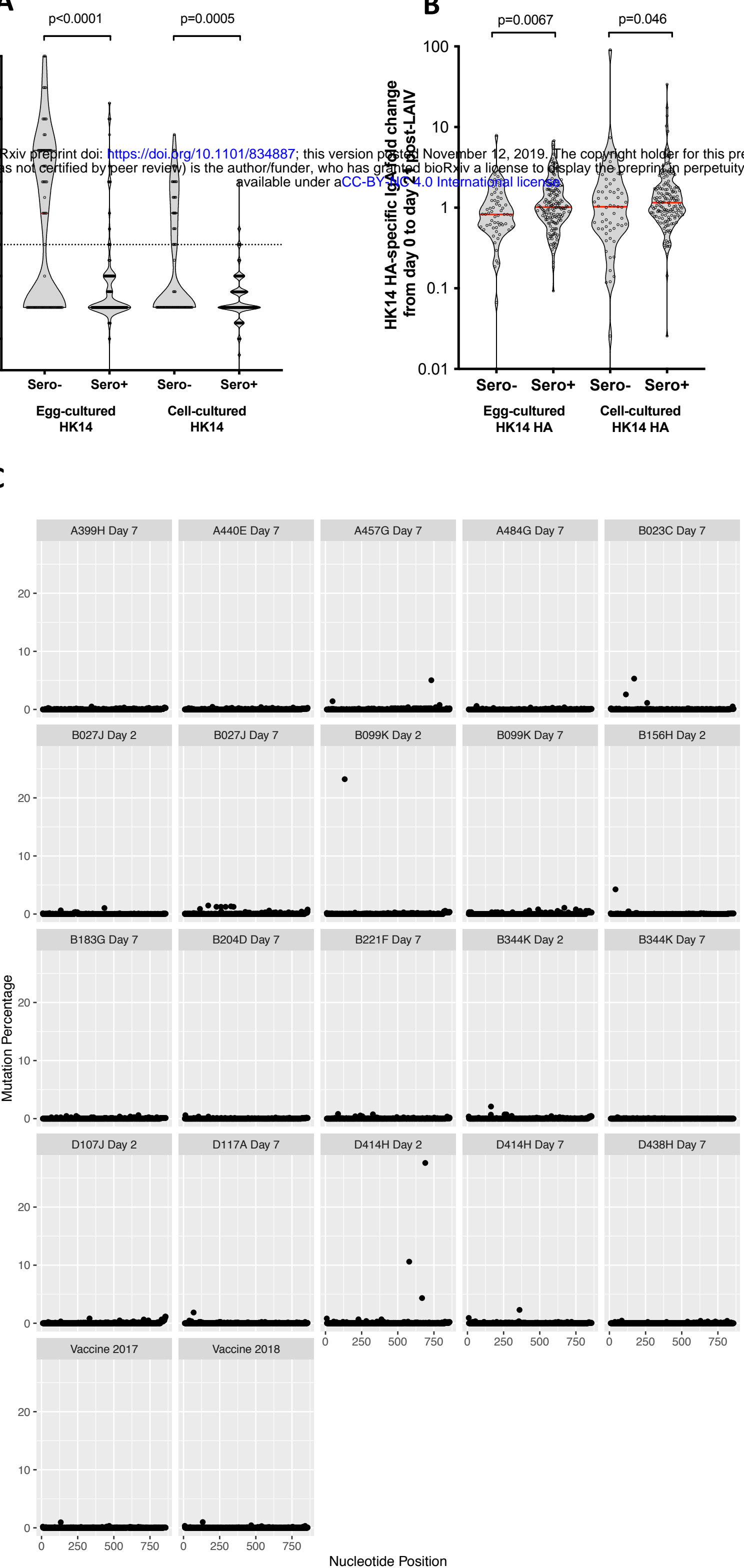